\title{
Delirium: the invisible syndrome
}

\author{
Paola Gnerre, ${ }^{1}$ Micaela La Regina, ${ }^{2}$ Chiara Bozzano, ${ }^{3}$ Fulvio Pomero, ${ }^{4}$ Roberta Re,${ }^{5}$ Michele Meschi, ${ }^{6}$ \\ Domenico Montemurro, ${ }^{7}$ Annalisa Marchetti, ${ }^{8}$ Mariangela di Lillo, ${ }^{9}$ Daniela Tirotta ${ }^{10}$
}

${ }^{1}$ Department of Internal Medicine, San Paolo Hospital, Savona; ${ }^{2}$ Department of Internal Medicine, POU del Levante Ligure, Asl 5 Liguria; ${ }^{3}$ Department of Internal Medicine, San Donato Hospital, Arezzo; ${ }^{4}$ Department of Internal Medicine, S. Croce e Carle Hospital, Cuneo; ${ }^{5}$ Department of Internal Medicine, Maggiore della Carità Hospital, Novara; ${ }^{6}$ Department of Internal Medicine, Santa Maria Hospital, Parma; ${ }^{7}$ Department of Internal Medicine, University Hospital of Padova; ${ }^{8}$ Department of Internal Medicine, National Institute for Elderly Care, Ancona; ${ }^{9}$ Department of Internal Medicine, Ospedali Riuniti, Marche Nord, Fano; ${ }^{10}$ Department of Internal Medicine, Cervesi Hospital, Cattolica (RN), Italy

\begin{abstract}
Delirium is a neuropsychiatric sindrome characterized by acute onset, a fluctuating course, an altered level of consciousness, disturbances in orientation, memory, attention, thinking, perception and behaviour. One third of patients aged 70 or older were admitted to the general medical service of an acute care hospital experience delirium. The development of delirium is associated with worse outcome increased a 10-fold risk for death and a 3- to 5-fold risk for nosocomial complications, prolonged length of stay, and greater need for nursing home placement after discharge. Therefore patients with delirium have higher morbidity and mortality rates, higher re-admission rates, and a greater risk of long term institutionalization care, thereby having a significant impact on both health and social care expenditure. The cost of delirium to the health-care system is then substantial. Despite its clinical importance and health-related costs, it often remains under-recognized and inadequately managed. Recent evidence suggests that a better understanding and knowledge of delirium among health care professionals can lead to early detection, the reduction of modifiable risk factors, and better management of the condition in the acute phase.
\end{abstract}

\section{Introduction}

The word delirium was first used as a medical term as easy as the first century AD to describe mental disorders associated with fever or head trauma. Currently, the term delirium is used to describe a cognitive disorder characterized by acute onset, a fluctuating course, an altered level of consciousness, and disturbances in orientation, memory, attention, thinking, perception and behavior. ${ }^{1}$ We distinguish the term delirium which indicates the neuropsychiatric syn-

Correspondence: Gnerre Paola, Department of Internal Medicine, San Paolo Hospital, via Genova I, 17100 Savona Italy. Tel.: +39.019.8404358 - Fax: +39.019.8404583.

E-mail: pgnerre@yahoo.it

Key words: Delirium; cognitive disorder; acute confusional state.

Received for publication: 24 July 2015.

Accepted for publication: 27 July 2015.

This work is licensed under a Creative Commons Attribution NonCommercial 4.0 License (CC BY-NC 4.0).

CCopyright P. Gnerre et al., 2016

Licensee PAGEPress, Italy

Italian Journal of Medicine 2016; 10:119-127

doi:10.4081/itjm.2016.626 drome from delusion a type of serious mental illness called a psychosis.

Delirium is extremely common but it is heterogenous in its precipitants, clinical manifestation, course and severity. The prevalence of delirium depends on the population that is being studied. The literature often reports different results. The rates of delirium in hospital admissions are from $11 \%$ to $42 \%$, while the incidence of delirium during a hospital stay ranges from $6 \%$ to $56 \%$. This broad range can be explained by patient inclusion criteria and different scoring methods for delirium. The overall prevalence of delirium in the community, residential and nursing home setting is just $1-2 \%$. This prevalence increases to 11 $24 \%$ in the setting of general hospital admission (prevalent cases). The incidence of delirium arising during a hospital stay ranges from $6 \%$ to as high as $56 \%$ (incident cases) and this incidence is even higher when more-specialized populations are considered, including those in postoperative, intensive-care, subacute and palliative-care setting. In fact, postoperative delirium occurs in $15-35 \%$ of surgical patients over the age of 65 . In the intensive care unit it occurs in 70$80 \%$ of elderly persons and is often a symptom that heralds the presence of life threatening conditions. ${ }^{2}$ Then, approximately, one third of patients aged 70 or older admitted to the general medical service of an acute care hospital experience delirium. In the hospital, delirium has been associated with a 10 -fold increased risk for death and with a 3 - to 5 -fold increased 
risk for nosocomial complications, prolonged length of stay, and greater need for nursing home placement after discharge. Therefore patients with delirium have higher morbidity and mortality rates, higher re-admission rates, and a greater risk of long term institutionalisation care, thereby having a significant impact on both health and social care expenditure. ${ }^{3}$ The cost of delirium to the health-care system is then substantial. Research in the USA indicates that hospital stays complicated by delirium account for approximately 1.5 million inpatient days, and \$US 6.9 billion in Medicare expenditure each year. Despite its clinical importance and health-related costs, it often remains under-recognized and inadequately managed. Recent evidence suggests that a better understanding and knowledge of delirium among health care professionals can lead to early detection, the reduction in modifiable risk factors, and better management of the condition in the acute phase. Many cases of delirium are potentially preventable, and primary and secondary care services should be taking active steps in order to prevent this condition. ${ }^{4}$ For this reason, many health care organizations have felt the need to produce guidelines for improving the management of delirium and to provide a systematic approach to recognizing, assessing, treating, and monitoring patients with delirium and acute problematic behavior.

\section{Etiology}

A common risk model for the development of delirium, recognized predisposing factors chronic that increase the vulnerability of the individual from precipitating factors acute that cause delirium. The presence of dementia (found in more than $2 / 3$ of the patients), advanced age, impaired visual acuity, use of urinary catheter, the physical restraint and hiring more than three drugs are the most important predisposing factors. ${ }^{5}$ The presence of a severe acute disease and/or infection, abstinence or alcohol intoxication are among the factors precipitating ${ }^{4}$ (Table 1). ${ }^{6}$ If more predisposing factors are in an individual, the higher is its vulnerability and minor precipitating factors are required for the development of the syndrome. ${ }^{5}$

\section{Clinical manifestation}

Patients with delirium have poor attention, memory disorders, speech disorder and frequent reversal of the sleep-wake cycle. In 1990 for the first time Lipowsky distinguished three subtypes of delirium in relation to the psychomotor state: a hypoactive form, a hyperactive form and a mixed form. Patients with hyperactive form are agitated, confused, sometimes aggressive and often have hallucinations. The patients with hypoactive form are apathetic and lethargic. They are frequently undiagnosed because erroneously considered depressed or demented. ${ }^{1}$ In the mixed form the patient shows features of both increased and decreased psychomotor activity. ${ }^{7}$ Delirium can be further classified into persistent, if it is continuous, and night if it occurs mainly in the late afternoon or at night. It is typical of patients with dementia and it is characterized by an alteration of the normal circadian rhythm.

\section{Diagnosis}

The diagnostic standard for delirium is taken from the fourth edition of the Diagnostic Manual of Mental Disorders of the American Society of Psychiatry ${ }^{3}$ (Figure 1). ${ }^{8}$ The diagnosis of delirium is placed in the presence of a reversible cognitive disorder, characterized by an acute onset (hours to days) and floating, a disorder of memory, attention, language, perception (visual and auditory hallucinations), of behavior and thought that occurs in the context of a medical condition (medical condition acute poisoning from substances or a withdrawal syndrome). ${ }^{3}$

The diagnosis of delirium can be accomplished smoothly through specific assessment scales. Among the best known rating scales studied for the diagnosis of delirium include: i) confusion assessment method (CAM) (Figure 2); ${ }^{6}$ ii) confusion assessment methodintensive care unit (CAM-ICU); iii) delirium rating scale (DRS); iv) delirium symptom interview; v) memorial delirium assessment scale (MDAS); vi) intensive care delirium screening checklist; vii) nursing delirium screening scale.

The CAM is between the scales the most effective and rapid with a sensitivity and specificity above $90 \%$. The scale DRS and the MDAS represent a tool to assess the degree of severity of delirium. ${ }^{8}$

A comprehensive history should confirm an acute change in cognitive function and must include information on intercurrent illness, medication use (including any modifications or taking some herbal products), alcohol withdrawal or recent environmental changes. It should also exclude conditions that mimic the delirium such as Alzheimer's disease, psychotic disorders and depression. Table $2^{1}$ shows the main clinical features that differentiate these disorders from delirium.

Physical examination and laboratory evaluation looking for any infectious diseases, metabolic disorders and endocrine, cardiovascular and cerebral diseases that could have caused the syndrome. ${ }^{1,4}$

Exogenous substances should be searched for suspected intoxication (drugs and narcotics). ${ }^{4}$ Computed tomographic scan and electroencephalogram will also help us to exclude respectively organic brain disease and a metabolic encephalopathy. Lumbar puncture should be performed in suspected meningitis. 


\section{Treatment}

The approach to the patient with delirium is the correction of all the factors potentially modifiable. ${ }^{1}$ It must, therefore, maintain the cardiovascular stability, a normal thermal curve, adequate oxygenation, a normal water balance, normal blood sugar levels and adequate caloric intake.

The treatment of delirium component consists of a non-drug and a drug. The drug component is the treatment of choice in patients with delirium and aims at the orientation of the patient. ${ }^{6}$

This includes maintaining the visual and tactile contact, the improvement of functional capacity and mobility and the use of clear and simple verbal instructions. Pre-existing visual and acoustic defects should be promptly corrected with the appropriate devices. We should minimize the noise and maintain a proper lighting (avoid excess light). It should avoid environmental changes and do not use means of restraint. It should also prevent the common complications related to hospitalization (falls, pressure ulcers, infections nosomiali, functional decline). ${ }^{4}$

Drug treatment of delirium should be reserved for the treatment of disease-causing delirium. However, if the patient presents agitated delusional and aggressive and non-drug measures alone are not able to control the clinical manifestation, the initiation of drug therapy is justified. The use of drugs is still recommended only for a short period (short-term treatment). ${ }^{9}$ For short-term treatment we mean a pharmacological treatment of a duration of one week or less. However to date the evidence based on the use of drugs in delirium is limited to a small number of studies (Table 3). ${ }^{1}$ Benzodiazepines are contraindicated in the treatment of delirium except delirium resulting in withdrawal crisis.

Table 1. Predisposing and precipitating factors of delirium.

\section{Predisposing factors of delirium}

Demographic characteristics (age $>65$ years, male sex)

Cognitive status (dementia and other causes of cognitive impairment, previous history of delirium, depression)

Visual and hearing impairment

Decreased oral intake (dehydration, malnutrition)

Drugs (treatment with multiple drugs, treatment with psychoactive drugs)

Alcohol abuse

Coexisting medical condition (multiple coexisting conditions as chronic renal, heart failure or hepatic disease, history of stroke, neurologic disease, metabolic derangements, fracture or trauma, terminal illness, infection with HIV illness)

Functional status (dependence, immobility, frailty, history of falls)

Pain

Constipation

Prolonged sleep deprivation

\section{Precipitating factors of delirium related to patient}

Intercurrent illnesses (infections, hypoxia and hypercapnia, severe acute illnesses for example, myocardial infarction, heart failure, renal or hepatic failure)

Urinary retention

Anemia

Constipation and fecal impaction

Fever shock

Iatrogenic complications

Metabolic derangements (electrolyte disturbances, glucose, acid-base balance)

Dehydration

Endocrine disease: hypo- or hyperthyroidism, addisonian crisis, hypopituitarism, hypo- and hyper parathyroidism

Vitamin deficiencies: thiamine, nicotinic acid, B12

Poor nutritional status

Neurological conditions (meningitis or encephalitis, cerebrovascular accident, subarachnoid haemorrhage, hypertensive encephalopathy, head trauma, epilepsy: complex partial seizures, post-ictal states, petit mal)

\section{Precipitating factors of delirium related to environment}

Environmental issues

Admission to intensive care unit

Environmental lighting

Sensory overload

\section{Precipitating factors of delirium related to procedures}

Use of physical restraints

Bladder catheterization

Surgery (orthopedic, cardiac surgery)

Hip fracture

Smoking cessation 


\section{Hospitalization criteria}

The decision to hospitalize a patient with suspected delirium requires the evaluation of multiple factors, in particular the timeliness of diagnostic evaluation, clinical stability and social support. Not all patients with delirium require hospitalization and also hospitalization may further exacerbate the clinical manifestation. ${ }^{4}$ The outpatient management may be indicated if the work-up diagnosis can be made quickly if the safety of the patient is not put at risk and if treatment of the triggers delirium is not complex (e.g., oral antibiotics for an uncomplicated urinary tract infection or side effects of a drug rapidly reversible upon discontinuation). It is also extremely important to the evaluation of the support and

\section{A. Disturbance of consciousness (i.e., reduced clarity of awareness of the environment) with reduced ability to focus, sustain or shift attention}

yes_no

B. A change in cognition or the development of a perceptual disturbance that is not better
accounted for by a preexisting, established or evolving dementia
yes_no

C. The disturbance develops over a short period of time (usually hours to days) and tends to fluctuate during the course of the day

yes__no

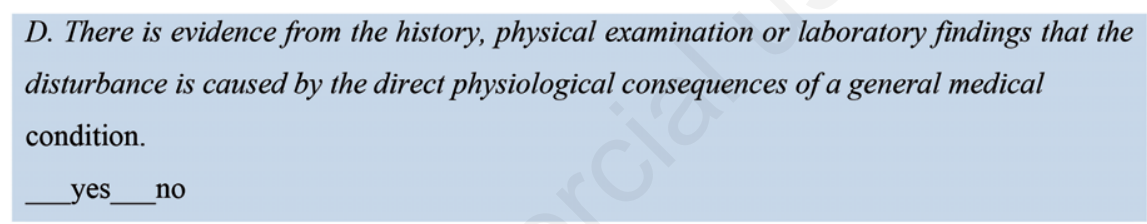

To make a diagnosis of delirium, the patient must have all four of the following criteria (AB-C-D)

Figure 1. Diagnostic criteria for delirium (DSM-IV).

Table 2. Differential diagnosis of delirium.

\begin{tabular}{|c|c|c|c|c|}
\hline Clinical features & Delirium & Dementia & Depression & Primary psychotic disorders \\
\hline Onset & Acute & Insidious & Acute or insidious & Acute or insidious \\
\hline Duration & Hours to weeks & Months to years & Weeks to months & Weeks to months \\
\hline Course & Fluctuating & Chronic and progressive & May be chronic & May be chronic \\
\hline Progress & Usually reversible & Irreversible & Usually reversibile & Usually reversible \\
\hline Level of consciousness & Altered & Usually clear & Clear & Clear \\
\hline Orientation & Variable & Disoriented & Oriented & Oriented \\
\hline Attention and concentration & Poor & Normal except in late stage & May be impaired & May be impaired \\
\hline Speech & Incoherent & Coherent until the late stage & Usually normal & May be pressured \\
\hline Thought & Process disorganized & Limited & Usually organized & May be disorganized \\
\hline Psychomotor activity & Variable & Normal & May be slow & Variable \\
\hline Perception & $\begin{array}{l}\text { Hallucinations are } \\
\text { frequent especially visual }\end{array}$ & $\begin{array}{c}\text { May have hallucinations } \\
\text { especially visual }\end{array}$ & $\begin{array}{c}\text { May have hallucinations } \\
\text { especially auditory }\end{array}$ & $\begin{array}{c}\text { May have hallucinations } \\
\text { especially auditory }\end{array}$ \\
\hline
\end{tabular}

Modified by Mittal et al., 2011. ${ }^{1}$ 
compliance of socio-family and residential location of the patient. In fact, in clinically stable patients it can be indicated an extra-hospital treatment. Hospitalization is required for all patients whose delirium is associated with an acute clinically destabilizing or in the event of non-family social support. The decision to hospitalize a patient must evaluate all the negative effects such as hospital inpatient complications and possible further worsening of symptoms of delirium following the change in the environment.

\section{The management of the patient with delirium: rationale and objectives}

Delirium is a common condition in medical patients (especially in elderly patients hospitalized for acute illness) but even so often unrecognized and untreated. Therefore the management of the patient with delirium should be a competence of internist.

This paper aims, in fact, at the following objectives:

\footnotetext{
Feature 1: Acute onset and fluctuating course

This feature is usually obtained from a family member or nurse and is shown by positive responses to the following questions:

Is there evidence of an acute change in mental status from the patient's baseline?

Did the (abnormal) behavior fluctuate during the day, that is, tend to come and go, or increase and decrease in severity?

Feature 2: Inattention

This feature is shown by a positive response to the following question:

Did the patient have difficulty in focusing attention, for example, being easily distractible, or having difficulty keeping track of what was being said?

Feature 3: Disorganized thinking

This feature is shown by a positive response to the following question:

Was the patient's thinking disorganized or incoherent, such as rambling or irrelevant conversation, unclear or illogical flow of ideas, or unpredictable switching from subject to subject?

Feature 4: Altered level of consciousness

This feature is shown by any answer other than alert to the following question:

Overall, how would you rate this patient's level of consciousness? [alert (normal), vigilant (hyperalert), lethargic (drowsy, easily aroused), stupor (difficult to arouse), or coma unarousable)]
}

The diagnosis of delirium by CAM requires the presence of features 1 and 2 and either 3 or 4 .

Figure 2. The Confusion Assessment Method (CAM) Diagnostic Algorithm.

Table 3. Drug therapy for delirium.

\begin{tabular}{|c|c|c|}
\hline Medication group & Dosage & Side-effects \\
\hline Typical antipsychotics: haloperidol & $\begin{array}{l}\text { Excessive agitation: } \\
0.25-1.0 \mathrm{mg} \text { PO BID/TID; } 0.25-1.0 \mathrm{mg} \text { PO or IM; } \\
\text { can be repeated every } 30-60 \mathrm{~min} \text { if needed }\end{array}$ & $\begin{array}{l}\text { Extrapyramidal symptoms, sedation, prolonged } \\
\text { QTc interval }\end{array}$ \\
\hline $\begin{array}{l}\text { Atypical antipsychotics } \\
\text { A. Risperidone: excessive agitation } \\
\text { B. Olanzapine: excessive agitation } \\
\text { C. Quetiapine: excessive agitation }\end{array}$ & $\begin{array}{c}\text { 0.25-0.5 mg PO BID/TID; } \\
\text { 0.25-0.5 mg PO; } \\
\text { can be repeated every 30-60 min if needed } \\
2.5-5.0 \mathrm{mg} \text { PO BID; } \\
2.5-5.0 \mathrm{mg} \text { PO or IM; } \\
\text { can be repeated every 30-60 min if needed } \\
25-50 \mathrm{mg} \text { BID/TID; } 25-50 \mathrm{mg} \text { PO; } \\
\text { can be repeated every } 30-60 \mathrm{~min} \text { if needed }\end{array}$ & $\begin{array}{c}\text { Metabolic dysfunction, extrapyramidal symptoms } \\
\text { hyperglycemia, prolonged QTc interval } \\
\text { As for risperidone } \\
\text { As for risperidone and olanzapine }\end{array}$ \\
\hline $\begin{array}{l}\text { Cholinesterase inhibitors: } \\
\text { Donepezil 5-10 mg }\end{array}$ & PO once daily & Gastrointestinal disturbances \\
\hline
\end{tabular}


i) to provide a practical tool for the prevention, detection, diagnosis and treatment of delirium, with a particular focus on the prevention of delirium in hospitalized patients considered at risk and the management of those subjects who develop delirium during hospitalization; ii) to help the physician to distinguish between the causes of delirium and to provide indications to identify patients with worse prognosis and the most appropriate care setting (outpatient, department of psychiatry, geriatrics, internal medicine, etc.). Delirium in children and teens is not the subject of this work.

\section{The management of the patient with delirium: methodology}

In order to provide evidence-based recommendations for the management of patients with malnutrition, we first verified the existence of guidelines on the matter. Therefore, we conducted a search using the following database guidelines: i) Scottish Intercollegiate Guidelines Network (SIGN); ii) Institute for Clinical Systematic Improvement (ICSI); iii) National Institute for Health and Clinical Excellence (NICE) - National Health System (NHS) evidence; iv) National Guideline Cleringhouse (NGC); v) Canadian Medical Association, CMA Infobase; vi) New Zealand Guidelines Group; vii) Italian National Health System Guidelines; viii) Clinical Practice Guidelines Portal; ix) eGuidelines.

The research was carried out by four authors independently, using as key-words the terms delirium, alcohol, HIV and AIDS when the site included the search function, and in other cases we list the last guidelines manually stored in the database or made reference to the mental illness. The results obtained separately were then compared and discussed together. The guidelines thus obtained were evaluated using the Appraisal of Guidelines, Research and Evaluation II (AGREE II) ${ }^{10}$ instrument by four authors independently. AGREE II assesses compliance with 23 requirements, meeting 6 domains as the explanation of the purpose, the clarity, the involvement of all stakeholders, the rigor of development, applicability and editorial independence of the same. Each author assessed the compliance of individual requirements with a score from 1 (disagree completely) to 7 (complete agreement). The scores assigned by each author were added within individual domains and reported with the highest and the lowest score possible within the domain based on the number of requirements included and the number of evaluators.

\section{The management of the patient with delirium: results}

Through the databases listed above, we identified 93 guidelines: 84 were excluded on the basis of the title or text or for other reasons and 9 were selected for evaluation. Of these, 6 face the general management of delirium in older people, one relies exclusively on nursing assessment of delirium, dementia and depression, one on the management of complications related to the use of alcohol and 1 on the management of delirium in patients with AIDS.

The overall quality of the guidelines selected was assessed by 2 authors (MLR and PG) separately using the AGREE instrument II. The evaluation results are shown in Table $4^{11-19}$ from which it follows that: i) with regard to the guidelines on the general management of delirium in older people, the guidelines produced by $\mathrm{NICE}^{11}$ have scored the most points ( 255 points) with good balance between the various domains; ii) the guidelines produced by the Clinical Epidemiology and Health Service Unit, Melbourne Health ${ }^{12}$ have appeared in the most severe expression of the objectives and motivations and methodology development, but with less clarity and less requirements applicability and editorial independence respected; iii) the guidelines produced by the Canadian Coalition for Seniors Mental Health ${ }^{13}$ have been shown to have clear objectives and motives, good clarity, but less rigorous in developing and less compliance with the requirements of applicability; iv) guidelines on nursing assessment of delirium, dementia and depression, produced by the Registered Nurses' Association of Ontario ${ }^{14}$ did just enough in all domains; v) guidelines on complications related to the use of alcohol, produced by NICE, ${ }^{15}$ proved to be of excellent quality in all domains evaluated; vi) the guidelines for the management of delirium in patients with HIV/AIDS, produced by the New York State Department of Health ${ }^{16}$ evaluation AGREE showed a clear expression of the objectives and motivations, good methodological rigor, discreet applicability and editorial independence; vi) guidelines on manangement of palliative care ${ }^{17}$ showed discreet clear expression but poor value in remaining domains; vii) the guidelines produced by American Medical Directors Association ${ }^{18}$ showed valid objective and motivations, clear expression but a low score in the domains of stakeholder involvement, rigor processing, applicability and editorial independece; viii) the guidelines produced by British Society of Geriatrics ${ }^{19}$ have good clarity, discrete targets and rigor in the processing but little value in the reimaning domains.

Based on the assessment AGREE, the guidelines produced by NICE $^{9}$ are, among the guidelines available, those of higher quality, whose implementation in clinical practice appears desirable.

\section{Clinical approach to patient with delirium}

The management of headache consisted of six: ${ }^{11}$ i) think delirium; ii) assessment of risk factors for delirium in all patients aged $>65$ years; iii) indicators 
of delirium: at hospital admission; iv) interventions to prevent delirium; v) diagnosis; vi) treatment.

The diagnostic algorithm shown in Figure 3 can help us to better handle the patient with delirium.

\section{Think delirium}

The internist should know that all those hospitalized or subjected to long-term hospitalization are at risk of delirium. Delirum is associated with worse outcomes (for example, an increased risk of dementia and/or death) and, for hospitalized patients, can prolong hospital stay and increase the risk of readmission.

\section{Assessment of risk factors}

Risk factors: age over 65 years, cognitive impairment (current or past) and/or dementia known, hip fracture, severe acute illness. If present, the patient is at risk of delirium. The patient should be carefully evaluated to search for changes in risk factors for delirium.

\section{Indicators of delirium: admission to the hospital}

Evaluate patients at risk of delirium with recent fluctuations or behavioral changes (in the order of hours or days) reported by themselves or by the care-giver. At- tention to behaviors indicative of hypoactive delirium. Behavioral changes occur as: i) cognitive function: deterioration of concentration, slowed responses, confusion; ii) perception: visual or auditory hallucinations; iii) physical function: reduced motility, agitation, changes in appetite, sleep disturbances; iv) social behavior: lack of cooperation in case of reasonable requests, interruption or alteration of communication, mood alterations.

\section{Interventions to prevent delirium}

Interventions to prevent delirium are the following: i) do not move patients between departments, unless it is absolutely necessary; ii) provide personalized assistance by a multi-disciplinary team trained and competent; iii) ensure appropriate lighting and a clear indication of the time (clock in ICU). A calendar should be easily visible to patients at risk; iv) provide stimulating cognitive activities (for example, the memory); v) facilitate visits of family and friends; vi) check dehydration and/or constipation; vii) improve cardiovascular stability, glycemic control and electrolyte balance, if necessary; viii) assessing the presence of hypoxia and optimize the oxygen saturation if necessary; ix) check infection by searching, avoid unnecessary catheterizations and maintain skin integrity;

Table 4. AGREE evaluation.

\begin{tabular}{|c|c|c|c|c|c|c|}
\hline & $\begin{array}{c}\text { Domain } 1 \\
\text { Motivation and } \\
\text { objective }\end{array}$ & $\begin{array}{c}\text { Domain } 2 \\
\text { Stakeholder } \\
\text { involvement }\end{array}$ & $\begin{array}{l}\text { Domain } 3 \\
\text { Rigor in } \\
\text { elaboration }\end{array}$ & $\begin{array}{l}\text { Domain } 4 \\
\text { Clarity of } \\
\text { exposition }\end{array}$ & $\begin{array}{c}\text { Domain } 5 \\
\text { Applicability }\end{array}$ & $\begin{array}{c}\text { Domain } 6 \\
\text { Editorial } \\
\text { independence }\end{array}$ \\
\hline $\begin{array}{l}\text { Clinical Epidemiology and } \\
\text { Health Service Unit, } \\
\text { Melbourne Health } \\
\text { Mel }^{2}\end{array}$ & $36 / 36$ & $34 / 36$ & $90 / 96$ & $44 / 48$ & $24 / 36$ & $16 / 24$ \\
\hline $\begin{array}{l}\text { Canadian Coalition for } \\
\text { Seniors' Mental Health }\end{array}$ & $35 / 36$ & $32 / 36$ & $73 / 96$ & $47 / 48$ & $25 / 36$ & $19 / 24$ \\
\hline $\begin{array}{l}\text { Department of Health and } \\
\text { Human Service, Tasmania }{ }^{17}\end{array}$ & $7 / 36$ & $2 / 36$ & $7 / 96$ & $26 / 48$ & $0 / 36$ & $6 / 24$ \\
\hline $\begin{array}{l}\text { American Medical } \\
\text { Director Association }^{18}\end{array}$ & $33 / 36$ & $6 / 36$ & $22 / 96$ & $20 / 48$ & $6 / 36$ & $6 / 24$ \\
\hline British Society of Geriatrics ${ }^{19}$ & $22 / 36$ & $16 / 36$ & $40 / 96$ & $38 / 48$ & $11 / 36$ & $0 / 24$ \\
\hline $\begin{array}{l}\text { Delirium: diagnosis, } \\
\text { prevention and } \\
\text { management }(\mathrm{NICE})^{11}\end{array}$ & $35 / 36$ & $34 / 36$ & $86 / 96$ & $48 / 48$ & $29 / 36$ & $20 / 24$ \\
\hline $\begin{array}{l}\text { Registered Nurses' } \\
\text { Association of Ontario }{ }^{14}\end{array}$ & $36 / 36$ & $19 / 36$ & $58 / 96$ & $37 / 48$ & $24 / 36$ & $12 / 24$ \\
\hline $\begin{array}{l}\text { Alcohol use disorders: } \\
\text { diagnosis and clinical } \\
\text { management of alcohol-related } \\
\text { physical complications (NICE) }{ }^{15}\end{array}$ & $36 / 36$ & $36 / 36$ & $89 / 96$ & $36 / 48$ & $35 / 36$ & $18 / 24$ \\
\hline $\begin{array}{l}\text { Cognitive disorders and } \\
\text { HIV/AIDS: HIV-associated } \\
\text { dementia and delirium, } \\
\text { New York State } \\
\text { Department of Health }{ }^{16}\end{array}$ & $34 / 36$ & $14 / 36$ & $75 / 96$ & $12 / 48$ & $24 / 36$ & $16 / 24$ \\
\hline
\end{tabular}


x) Prevent hypo- and immobility by encouraging patients to move; xi) control pain by evaluating and researching non-verbal signs of pain, especially in those with communication difficulties; xii) revise drug therapy in people taking numerous medications; xiii) check any nutritional deficiency; xiv) check sensory deficits resolving any reversible cause of sensory deficit, such as plugs of cerumen, ensuring that patients use their hearing aids and vision; $\mathrm{xv}$ ) promote correct patterns of sleep hygiene avoiding medical procedures or nursing during the night hours and planning therapies not to disturb sleep at night.

\section{Diagnosis}

Once the indicators of delirium have been identified in hospitalized patients, perform a clinical assessment based on the criteria of the Diagnostic and Statistical Manual of mental disorders $4^{\text {th }}$ ed. (DSMIV) (Table 1) or with short CAM (Table 2) to confirm the diagnosis. In critical care or in the recovery room, use the CAM-ICU.

\section{Treatment of delirium}

Identify and treat the possible cause and/or combi-

Are there the following risk factors?

Age $>65$ years

Impairment of cognitive function and/or dementia

Hip fracture

Severe acute illness

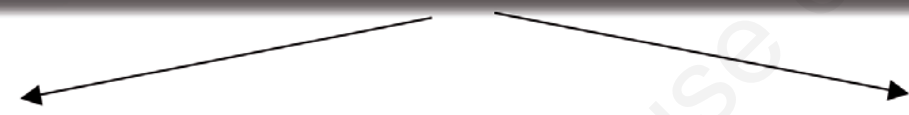

Yes

No

Patient at risk

of delirium

Patient is not at

risk of delirium

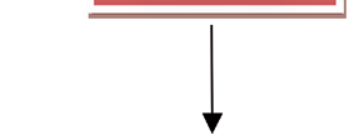

Are there indicators of delirium?

There are changes (in the order of hours or days) in cognitive

functions, perception and behavior
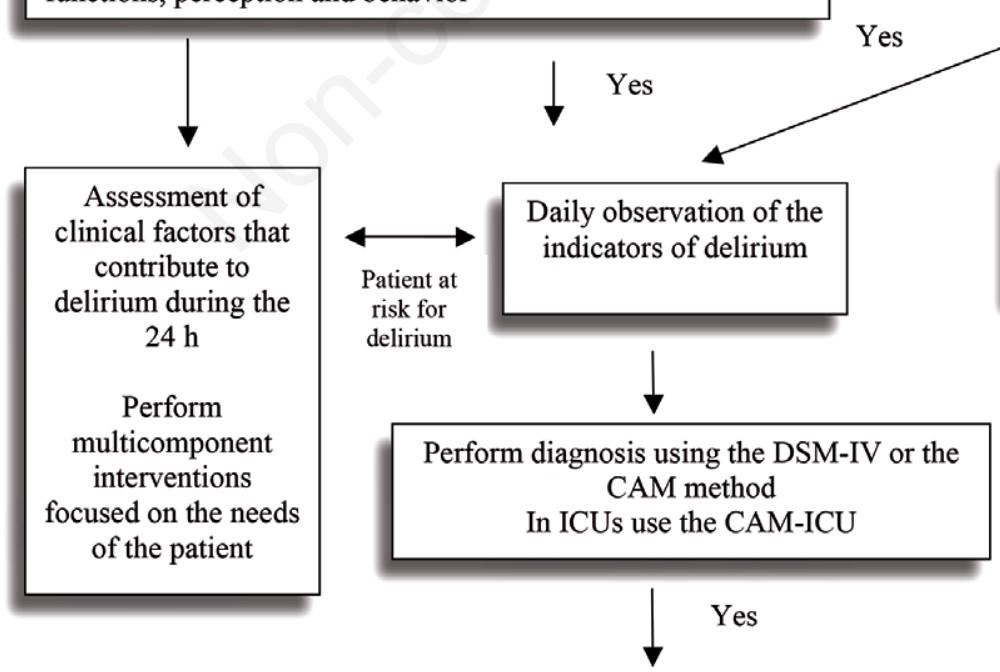

Is delirium diagnosed? If it is difficult to

distinguish between delirium, dementia and

delirium with dementia treat delirium first

Daily observation of the indicators of delirium

Figure 3. Diagnostic algorithm of delirium. 
nation of underlying causes. Ensure effective communication, try to restore the orientation of the subject and reassure him. Engage family, friends and care-givers in this goal. If a patient with delirium is in distress or is considered risky for himself or for others, and verbal and non-verbal de-escalation techniques are ineffective or inappropriate, consider a short course (usually a week or less) therapy with haloperidol or olanzapine. Start with the lowest possible dose and titrate with caution according to symptoms. A baseline electrocardiogram is recommended prior to starting treatment.

The initial dose of haloperidol is between 0.25 and $0.50 \mathrm{mg}$ twice a day.

Use the anti-psychotic with caution or not at all in patients with diseases such as Parkinson's disease or dementia with Lewy bodies.

\section{Conclusions}

Delirium is a medical condition that requires a holistic and integrated approach to the patient. Holistic because many factors (pathological, social and environmental) determine its development and integrated because the correct approach is requiring multidisciplinary collaboration of doctors, nurses and care givers.

Concluding the diagnosis and treatment of delirium, especially in the elderly, it is an important skill for the internist and nurse in internal medicine and NICE guidelines can be a valuable support to good practice.

Despite its clinical importance, delirium remains a disease that often goes undiagnosed and therefore untreated, especially in its underactivity. This is even more important, if you think that the delirium would be preventable in more than a third of the cases. ${ }^{5}$ Therefore, we could hypothesize that delirium can be considered as an indicator of quality of care provided by the NHS, particularly for the care of older patients. $^{8}$

\section{References}

1. Mittal V, Muralee S, Williamson D, et al. Delirium in the elderly: a comprehensive review. Am J Alzhaimer Dis 2011;26:97-109.

2. Morandi A, Pandharipande P, Trabucchi M, et al. Understanding international difference in terminology of delirium and other types of acute brain dysfunction in critically ill patients. Intens Care Med 2008;34:1907-15.

3. American Psychiatric Association. Diagnostic ad statistical manual of mental disorders, 4th ed. Washigton, DC: America Psychiatric Association; 1994.

4. Marcantonio ER. In the clinic delirium. Ann Intern Med 2011;ITC:6-16.

5. Rizzo JA, Bogardus ST Jr, Leo-Summers L, et al. Mulicomponent targeted intervention to prevent delirium in hospitalized older patients: What is the economic value?. Med Care 2001;39:740-52.

6. Fong TG, Tulebaev SR, Inouye SK. Delirium in the elderly adults: diagnosis, prevention and treatment. Nat Rev Neurol 2009;5:210-20.

7. Inouye SK. Delirium in older persons. N Engl J Med 2006;354:1157-65.

8. Saxena S, et al. Delirium in the elderly: a clinical review. Postgrad Med J 2009;85:405-13.

9. Tropea J, Slee JA, Brand CA, et al. Clinical practice guidelines for the management of delirium in older people in Australia. Austral J Ageing 2008;27:150-6.

10. AGREE Next Steps Consortium. The AGREE II Instrument [Electronic version]; 2009. Available from: http:// www.agreetrust.org

11. National Institute for Health and Clinical Excellence (NICE). Delirium: diagnosis, prevention and management; NICE guidelines [CG103]; published: July 2010. Available from: https://www.nice.org.uk/guidance/cg103

12. Clinical Epidemiology and Health service Unit; Melbourne Health; the Delirium Clinical Guideline Expert Working Group. Clinical practice guidelines for the management of delirium in older people; 2006. Available from: http://www.health.gov.au/internet/main/publishing.nsf/Content/delerium-guidelines.htm

13. Canadian Coalition for Seniors' Mental Health. National guidelines for seniors' mental health: the assessment and treatment of delirium; 2006. Available from: http://www. ccsmh.ca/en/natlGuidelines/delirium.cfm

14. Registered Nurses' Association of Ontario. Screening for delirium, dementia and depression in the older adults; 2003. Available from: http://rnao.ca/bpg/guidelines/screening-delirium-dementia-and-depressionolder-adult

15. National Institute for Health and Clinical Excellence (NICE). Alcohol use disorders: diagnosis and clinical management of physical alcohol-related complications; NICE guidelines [CG100]; published: June 2010. Available from: https://www.nice.org.uk/guidance/cg100

16. New York State Department of Health. Cognitive disorders and HIV/AIDS: HIV-associated dementia and delirium; 2007. Available from: http://www.hivguidelines. org/clinical-guidelines/hiv-and-mental-health/cognitivedisorders-and-hiv-aids/

17. Tasmania Deptartment of Health and Human Services. Palliative care - Care management guidelines: delirium; 2009. Available from: http://www.dhhs.tas.gov.au/palliativecare/health_professionals/symptom_management_guidelines

18. American Medical Directors Association (AMDA). Delirium and acute problematic behavior in the longterm care setting. Columbia (MD): American Medical Directors Association (AMDA); 2008. Available from: https://www.nhqualitycampaign.org/files/Excerpts_fro m_AMDA_Clinical_Guidelines.pdf

19. British Society of Geriatrics. Guidelines for the prevention, diagnosis and management of delirium in older people in hospital; 2005. Available from: http://www. bgs.org.uk/index.php/clinicalguides/170-clinguidedeliriumtreatment 